\title{
Pathogenicity of Peptostreptococcus micros morphotypes and Prevotella species in pure and mixed culture
}

\author{
P. J. VAN DALEN*, E. C. VAN DEUTEKOM-MUldER, J. DE GRAAFF and T. J. M. VAN STEENBERGEN \\ Department of Oral Microbiology, Academic Centre for Dentistry Amsterdam, v.d. Boechorststraat 7, 1081 BT \\ Amsterdam, The Netherlands
}

\begin{abstract}
Recently, an atypical rough colony morphotype of Peptostreptococcus micros, a species which is found in ulcerating infections, including periodontitis, was isolated. The virulence of morphotypes alone and in combination with Prevotella intermedia and $\boldsymbol{P}$. nigrescens was investigated both in vivo and in vitro. All strains tested induced abscesses containing fluid pus in a mouse skin model, and lesions caused by monocultures of the rough morphotype strains of $\boldsymbol{P}$. micros were statistically significantly larger than those induced by the smooth morphotype strains. Inocula containing both morphotypes produced similar sized abscesses compared to mono-inocula containing the same bacterial load. Both Prevotella species induced small abscesses when inoculated alone, and when Pr. nigrescens was inoculated with one of the other strains, the abscesses were not significantly different from the abscesses induced by the mono-infections of this strain. Synergy, in terms of higher numbers of colony forming units (cfu) in the mixed inocula, was found for all combinations of the rough morphotypes of $P$. micros and both Prevotella spp. Pus from abscesses caused by combinations of Peptostreptococcus and Prevotella spp. transmitted the infection to other mice, but no abscesses were formed in mice inoculated with pus induced by mono-inocula. These results demonstrated synergic activity between both rough and smooth $P$. micros strains and oral Prevotella strains. The in-vitro co-culture experiments produced no evidence of growth stimulation. The effect of $P$. micros strains on the immune system was investigated by testing their ability to initiate luminol-dependent chemiluminescence of polymorphonuclear leucocytes in the presence and absence of human serum. In the latter, the rough morphotype strains initiated higher counts than the smooth morphotype strains. Further work is needed to elucidate the difference in virulence between the smooth and the rough morphotype cells of $P$. micros and the nature of the interaction with the Prevotella spp.
\end{abstract}

\section{Introduction}

Gram-positive anaerobic cocci are commonly found on the mucous membranes of the oral cavity, the intestines and the vagina. Frequently, the isolated strains of this group belong to the genus Peptostreptococcus. $P$. micros is considered an important pathogen in the aetiology of mixed anaerobic infections, including periodontitis, and is usually absent or present in low numbers in the plaque of healthy individuals $[1,2]$. A previous study described the existence of a rough and a

Received 28 Jan. 1997; revised version accepted 12 June 1997.

Corresponding author: Dr T. J. M. van Steenbergen.

* Present address: TNO Prevention and Health, Division of Immunological and Infectious Diseases, PO Box 2215, 2301 CE Leiden, The Netherlands. smooth morphotype of $P$. micros, which differ with respect to the presence of fibrillar structures on the cell wall, the composition of cell wall proteins, hydrophobic activity and ability to lyse erythrocytes [3].

Although two genogroups of Prevotella intermedia were described previously $[4,5]$ it is now accepted that these comprise two distinct species Pr. intermedia and $P r$ nigrescens [6]. Both species have been isolated from plaque samples from patients with periodontitis [7, 8]. Sundqvist et al. described the importance of black pigmented anaerobic rods in mixed infections with oral bacteria, and hypothesised that $P$. micros may be essential to induce transmissible abscesses [9]. The aim of this study was to investigate the pathogenicity of $P$. micros and its behaviour in mixed infections with Pr. intermedia and Pr. nigrescens. An in-vivo mouse subcutaneous skin model was selected as one of the 
parameters for pathogenicity $[10,11]$. Nutritional interactions between $P$. micros, Pr. intermedia and Pr. nigrescens strains were investigated in co-culture experiments in various fluid media and on solid media, and polymorphonuclear leucocyte (PMNL) activity induced by $P$. micros cells was assessed by measuring luminol-dependent chemiluminescence.

\section{Materials and methods}

\section{Bacterial strains, culture and identification}

Bacterial strains were grown anaerobically $\left(\mathrm{N}_{2} 80 \%\right.$, $\mathrm{CO}_{2} 10 \%, \mathrm{H}_{2} \quad 10 \%$ ) for 4 days on blood agar plates (Oxoid No. 2 agar, supplemented with defibrinated horse blood $5 \%$, haemin $5 \mathrm{mg} / \mathrm{L}$ and menadione $1 \mathrm{mg} / \mathrm{L}$ at $37^{\circ} \mathrm{C}$, unless stated otherwise). In monoinfections of $P$. micros, five strains of the smooth and five strains of the rough morphotype were employed. In the mixed inocula one strain of each morphotype of $P$. micros and one strain of each species of Prevotella were used. $P$. micros strain ATCC $33270^{\mathrm{T}}$ represented the smooth morphotype; HG 1467, isolated from the periodontal pocket of a patient with periodontitis, was the representative of the rough morphotype of $P$. micros. The identification of Pr. intermedia ATCC $25611^{\mathrm{T}}$, isolated from an empyema sample, and $P r$. nigrescens $\mathrm{HG} 187$, isolated from a patient with periodontitis, was based on colony morphology, physiological characteristics, production of black pigment on horse blood agar and DNA-DNA re-association studies [5, 6]. Enzyme electrophoresis of malate and glutamate dehydrogenase was performed to confirm the differentiation between $\mathrm{Pr}$. intermedia and $\mathrm{Pr}$. nigrescens [4]. After culture on blood agar for 2 days, about 20 colonies of each species were harvested and washed in $1 \mathrm{ml}$ of $0.1 \mathrm{M}$ Tris- $\mathrm{HCl}(\mathrm{pH} \mathrm{7.5).} \mathrm{The}$ suspensions were sonicated on ice with an amplitude of $20 \mu \mathrm{m}$ for $30 \mathrm{~s}$ and centrifuged for $10 \mathrm{~min}$ at $14000 \mathrm{rpm}$. The supernate was centrifuged once again and the cleared supernate was applied on a native polyacrylamide $(10-15 \%)$ gel and electrophoresed in the PhastSystem (Pharmacia). The running conditions were (1.1) $400 \mathrm{~V}, 10 \mathrm{~mA}, 2 \mathrm{~W}, 15^{\circ} \mathrm{C}, 10 \mathrm{Vh}$; (1.2, sample applicator down) $400 \mathrm{~V}, 0.1 \mathrm{~mA}, 2 \mathrm{~W}, 15^{\circ} \mathrm{C}$, $2 \mathrm{Vh}$; (1.3, sample applicator up) $400 \mathrm{~V}, 10 \mathrm{~mA}, 2 \mathrm{~W}$, $15^{\circ} \mathrm{C}, 125 \mathrm{Vh}$. The gels were stained according to Shah et al. [4] with a modified $\mathrm{pH}$ of 7.0 for glutamate dehydrogenase; L-malic acid (Sigma, cat. no. M1000) and DL-glutamic acid (Sigma, cat. no. G1126) were employed respectively as substrates. Identification of $P$. micros strains was based on Gram's stain, anaerobic growth, DNA-DNA homology [3] and biochemical reactions with the ATB $32 \mathrm{~A}$ and API-ZYM kits (Analytab Products, Montalieu-Vercieu, France).

\section{Experimental mouse model}

The mouse model was performed essentially as described by van Steenbergen et al. [11]. Bacteria were harvested from blood agar plates, washed, suspended in Schaedler Broth (BBL Microbiology Systems, Cockeysville, MD, USA) and adjusted to $5 \times 10^{9} \mathrm{cfu} / \mathrm{ml}$. This concentration was chosen to induce abscesses [9] but not to kill mice (LD50 for $P$. micros is $4.2 \times 10^{10} \mathrm{cfu}$ [12]). The total bacterial load per inoculum was $5 \times 10^{8}$ cfu in all experiments. Care was taken to maintain anaerobic conditions throughout the preparation of the suspensions. To compare the virulence of the smooth and rough morphotype, five strains of each type were tested. In the experiments with mixed inocula, $P$. micros ATCC $33270^{\mathrm{T}}$ and HG 1467, Pr. intermedia ATCC $25611^{\mathrm{T}}$ and Pr. nigrescens HG 187 were investigated. Two mice (Swiss) were inoculated with each strain or combination of strains. All animal experiments were performed twice with independent batches of bacteria and mice. The back of each mouse was inoculated with two $100-\mu \mathrm{l}$ volumes of the requisite bacterial suspension, $c .1 \mathrm{~cm}$ from the mid-line. Sterile broth was used as the control inoculum. Each day, all mice were examined for clinical signs and after 4 days they were killed by cervical dislocation. Skin fragments were excised and the largest diagonal of the abscess was measured with vernier callipers. In the mixed infections, the largest diagonal and the diagonal perpendicular to this were measured [10]. To determine transmissibility, the contents of two abscesses, one from each of two mice, were collected with a sterile cotton swab and pooled in $400 \mu$ l of sterile phosphatebuffered saline (PBS). This suspension was injected into the back of one mouse as described above. The two other abscesses, one from each mouse, were excised, homogenised aseptically in a grinder in $5 \mathrm{ml}$ of sterile PBS, serially diluted in ten-fold steps and inoculated on to blood agar plates. Viable counts were determined after growth for $96 \mathrm{~h} ; P$. micros (rough and smooth morphotypes) and Prevotella spp. were counted on the basis of colony morphology, but Pr. intermedia and Pr. nigrescens could not be distinguished.

In a separate set of experiments, abscess formation by both mono-infections and mixed bacterial inocula was examined histologically. The skin fragment containing the abscess was excised, stretched on a sheet of cork to avoid deformation and fixed in $4 \%$ buffered formalin for at least $24 \mathrm{~h}$ at room temperature. After fixation, the fragments were embedded in paraffin wax and $5-\mu \mathrm{m}$ thick sections were cut from the centre of the lesions, stained with haematoxylin and eosin and examined microscopically. Two mice were used for each strain or combination and experiments were performed twice with independent batches of bacteria and mice.

\section{Co-cultures in broth}

To study synergic growth of the morphotypes of $P$. micros and the Prevotella spp., bacteria were harvested from blood agar plates into BM3 broth (trypticase soy broth $10 \mathrm{~g} / \mathrm{L}$, proteose peptone $10 \mathrm{~g} / \mathrm{L}$, yeast extract 
$5 \mathrm{~g} / \mathrm{L}, \mathrm{NaCl} 5 \mathrm{~g} / \mathrm{L}, \mathrm{K}_{2} \mathrm{HPO}_{4} 5 \mathrm{~g} / \mathrm{L}, \mathrm{pH}$ 7.4) and washed in the same medium. The bacterial pellets were resuspended and adjusted to an $\mathrm{OD}_{650}$ of 0.5 $\left(5 \times 10^{7} \mathrm{cfu} / \mathrm{ml}\right)$ in BM3 broth. For each strain or combination of strains, three Coleman tubes (Coleman, USA), containing $20 \mathrm{ml}$ of BM3 broth, were inoculated with $100 \mu \mathrm{l}$ of these suspensions. Each strain was cultured in monoculture and in co-culture with one of the other strains. The $\mathrm{OD}_{650}$ of all cultures was measured twice a day for 4 days, in a Perkin-Elmer Junior III spectrophotometer.

\section{Chemiluminescence}

Chemiluminescence experiments were performed as described previously $[13,14]$. Essentially, $5 \times 10^{6} \mathrm{cfu}$ of $P$. micros were mixed with $5 \times 10^{5}$ PNMLs. As controls for the PMNL activity, $80 \mathrm{mM}$ PMA (phorbol 12-myristate 13-acetate; Sigma) in DMSO:buffered physiological saline 1:1 (5 $\mu \mathrm{l})$ and a reaction mixture without bacteria were used. Luminol-dependent chemiluminescence was measured in the presence or absence of pooled human serum $5 \%$ for $5 \mathrm{~s}$ in a Lumacounter (Lumac, model 2080) at $\mathrm{t}=0,10,20$ and $30 \mathrm{~min}$. Results of $t=10-30 \mathrm{~min}$ of each experiment were averaged and transformed to a percentage of the PMA control. Throughout the experiments the PMNLs were collected from the same donor and the same batch of pooled human serum was used in all experiments. All reactions were performed in duplicate in one experiment. The experiments with serum were repeated seven times and the experiments without serum on four occasions.

\section{Statistical analysis}

All results were analysed statistically by a two-tailed Mann-Whitney U test.

\section{Results}

\section{Experimental mouse model}

Abscess formation in mice was examined daily for $>2$ weeks and during the study no mice died or showed signs of illness. Although the smooth and the rough morphotype strains of $P$. micros varied in their ability to form abscesses sterile broth induced no visible macroscopic reaction. After 4 days the abscesses reached their maximum size; the mean length of the longest diagonal of the abscesses caused by the smooth and rough morphotype strains was 4.5 (SD 1.9) $\mathrm{mm}$ and 5.4 (SD $0.9) \mathrm{mm}$ respectively $(\mathrm{p}<0.05)$ (data not shown). In a second set of experiments single species and mixed inocula of each of the morphotypes of $P$. micros, and of $P r$. intermedia and Pr. nigrescens were administered. All strains formed abscesses containing fluid pus. The mean surface area of the abscesses induced by mixed inocula was slightly larger than that of the abscesses induced by mono-inocula but these differences were not statistically significant (Table 1). When cells of both morphotypes of $P$. micros were combined, the number of cfu of the smooth morphotype strain decreased, whereas the recovery of the rough morphotype strain remained the same. When peptostreptococci were combined with one of the Prevotella species, the numbers of cfu of both P. micros and Prevotella were much higher than those recovered from the mono-inocula (Table 2). This effect

Table 1. Size of abscesses induced in mice by $P$. micros, $P r$, intermedia and Pr. nigrescens, alone and in mixed infection

\begin{tabular}{|c|c|c|c|c|c|}
\hline \multirow[b]{2}{*}{ Species } & \multirow[b]{2}{*}{ Strain } & \multicolumn{4}{|c|}{ Mean (SD) surface area $\left(\mathrm{mm}^{2}\right)$ of abscesses with strain combination } \\
\hline & & $\begin{array}{c}\text { P. micros } \\
\text { (smooth) } \\
\text { ATCC } 33270^{\mathrm{T}}\end{array}$ & $\begin{array}{l}P . \text { micros } \\
\text { (rough) } \\
\text { HG } 1467\end{array}$ & $\begin{array}{l}\text { Pr. intermedia } \\
\text { ATCC } 25611^{\mathrm{T}}\end{array}$ & $\begin{array}{c}\text { Pr. nigrescens } \\
\text { HG } 187\end{array}$ \\
\hline P. micros (smooth) & ATCC $33270^{\mathrm{T}}$ & $27(0)^{*}$ & $29(6)$ & $41(13)$ & $48(12)$ \\
\hline P. micros (rough) & HG 1467 & - & $33(9)$ & $42(12)$ & 47 (11) \\
\hline Pr. intermedia & ATCC $25611^{\mathrm{T}}$ & - & - & $43(20)$ & $41(14)$ \\
\hline Pr. nigrescens & HG 187 & - & - & - & $32(12)$ \\
\hline
\end{tabular}

*Figures on the diagonal represent the data from monocultures

Table 2. Recovery of bacteria from primary abscesses in mice induced by $P$. micros strains ATCC $33270^{\mathrm{T}}$ (smooth morphotype) and HG 1467 (rough morphotype), Pr. intermedia strain ATCC $25611^{\mathrm{T}}$ and Pr. nigrescens strain HG 187, alone and in mixed infection

\begin{tabular}{|c|c|c|c|c|c|c|}
\hline \multirow[b]{2}{*}{ Species } & \multirow[b]{2}{*}{ Strain } & \multicolumn{5}{|c|}{ Number of cfu recovered with strain combination } \\
\hline & & None & $\begin{array}{c}\text { P. micros } \\
\text { (smooth) } \\
\text { ATCC } 33270^{\mathrm{T}}\end{array}$ & $\begin{array}{c}P \text { micros } \\
\text { (rough) } \\
\text { HG } 1467\end{array}$ & $\begin{array}{l}\text { Pr. intermedia } \\
\text { ATCC } 25611^{\mathrm{T}}\end{array}$ & $\begin{array}{c}\text { Pr. nigrescens } \\
\text { HG } 187\end{array}$ \\
\hline P. micros (smooth) & ATCC $33270^{\mathbf{T}}$ & $2 \times 10^{7}$ & - & $2 \times 10^{6}$ & $5 \times 10^{7}$ & $3 \times 10^{7}$ \\
\hline P. micros (rough) & HG 1467 & $2 \times 10^{6}$ & $1 \times 10^{6}$ & - & $8 \times 10^{7}$ & $9 \times 10^{7}$ \\
\hline Pr. intermedia & ATCC $25611^{\mathrm{T}}$ & $9 \times 10^{5}$ & $6 \times 10^{7}$ & $1 \times 10^{8}$ & - & nd \\
\hline Pr. nigrescens & HG 187 & $5 \times 10^{7}$ & $2 \times 10^{8}$ & $3 \times 10^{8}$ & nd & - \\
\hline
\end{tabular}

nd, no distinction could be made between Pr. intermedia and Pr. nigrescens based on colony morphology.

Inoculum size, $5 \times 10^{8} \mathrm{cfu}$. 
was maximal for the combination of Pr. intermedia with $P$. micros (rough morphotype) in which the numbers of cfu of $\mathrm{Pr}$. intermedia were 220 and 80 times higher, respectively compared to mono-inocula. No change in the number of cfu of either morphotype of $P$. micros occurred when they were inoculated singly or together (Table 2). The number of rough P. micros cells increased 80-100 times when combined with either of the Prevotella spp. in contrast with the smooth morphotype which remained stable in mixed culture. The number of cfu of Pr. nigrescens increased in mixed inocula with both $P$. micros strains, although to a lesser extent than for Pr. intermedia. When the pus from these abscesses was inoculated into other mice, the bacteria in pus derived from mono-inocula did not survive, whereas bacteria in the mixed inocula remained viable ( $P$. micros was recovered at $10^{3}-10^{5} \mathrm{cfu}$ and the Prevotella spp. reached a concentration equal in number to that in the primary abscesses). When both morphotypes of $P$. micros were combined, very low viable counts were recovered from the abscesses produced by pus inoculated into other mice (Table 3). Histologically, the abscesses consisted of a central collection of pus surrounded by a fibrous capsule; the outermost part of the lesion featured a distinct band of acute inflammatory cells. No differences were found in the histological samples of the abscesses induced by the different inoculations.

\section{Co-cultures in broth}

Monocultures in BM3 broth showed a characteristic growth curve (Fig. 1). The P. micros strains grew more slowly than $P r$ intermedia, reaching the stationary phase at c. $50 \mathrm{~h}$, without exceeding an $\mathrm{OD}_{650}$ of 0.2 . Pr. intermedia grew relatively rapidly and reached

Table 3. Recovery of bacteria from abscesses transmitted from one mouse to another, induced by $P$. micros strains ATCC $33270^{\mathrm{T}}$ (smooth morphotype) and HG 1467 (rough morphotype), Pr. intermedia strain ATCC $25611^{\mathrm{T}}$ and $P r$. nigrescens strain HG 187 , alone and in mixed infection

\begin{tabular}{|c|c|c|c|c|c|c|}
\hline \multirow[b]{2}{*}{ Species } & \multirow[b]{2}{*}{ Strain } & \multicolumn{5}{|c|}{ Number of cfu recovered with strain combination } \\
\hline & & None & $\begin{array}{c}\text { P. micros } \\
\text { (smooth) } \\
\text { ATCC } 33270^{\mathrm{T}}\end{array}$ & $\begin{array}{l}P \text { micros } \\
\text { (rough) } \\
\text { HG } 1467\end{array}$ & $\begin{array}{l}\text { Pr. intermedia } \\
\text { ATCC } 25611^{\mathrm{T}}\end{array}$ & $\begin{array}{c}\text { Pr. nigrescens } \\
\text { HG } 187\end{array}$ \\
\hline P. micros (smooth) & ATCC $33270^{\mathrm{T}}$ & 150 & $\ldots$ & $3 \times 10^{3}$ & $4 \times 10^{7}$ & $3 \times 10^{7}$ \\
\hline P. micros (rough) & HG 1467 & 0 & $1 \times 10^{3}$ & - & $1 \times 10^{5}$ & $8 \times 10^{7}$ \\
\hline Pr. intermedia & ATCC $25611^{\mathrm{T}}$ & 0 & $5 \times 10^{7}$ & $4 \times 10^{7}$ & - & nd \\
\hline Pr: nigrescens & HG 187 & 0 & $1 \times 10^{8}$ & $2 \times 10^{8}$ & nd & - \\
\hline
\end{tabular}

nd, no distinction could be made between Pr. intermedia and Pr. nigrescens based on colony morphology.

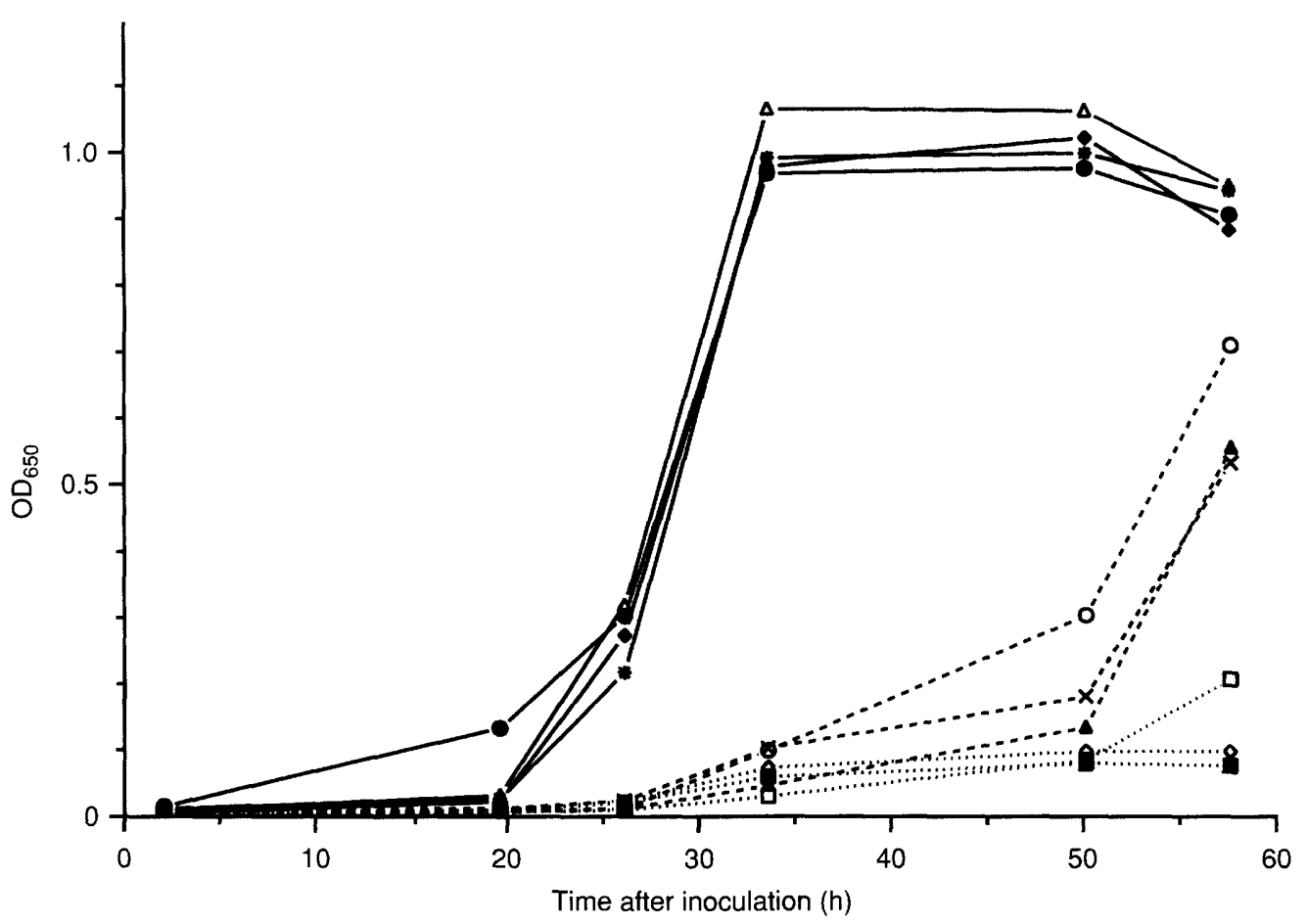

Fig. 1. Growth in broth of P. micros ATCC 33270 (smooth) (Pms) and HG 1467 (rough) (Pm r), Pr. intermedia ATCC 25611 (Pri) and Pr. nigrescens HG $187(\operatorname{Pr} \mathrm{n})$ in pure and mixed cultures: $\triangle-\triangle, \operatorname{Pr} \mathrm{i}, *-*, \operatorname{Pr} \mathrm{i}+\operatorname{Pr} \mathrm{n} ; \mathbf{-}-\mathbf{0}, \operatorname{Pm} \mathrm{r}+$

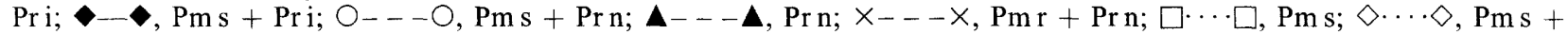
Pm r; $\mathbf{D}, \mathrm{Dm}$. 
stationary phase at about $35 \mathrm{~h}$ while $\operatorname{Pr}$. nigrescens failed to reach this stage of growth within 4 days. The growth curve for mixtures of peptostreptococci with one of the black pigmented species was similar to that of the Prevotella spp. in mono-culture, the addition of a $P$. micros strain did not influence this pattern.

\section{Chemiluminescence}

Chemiluminescence experiments were performed on both morphotypes of $P$. micros, with and without the addition of pooled human serum. In the latter experiments the smooth morphotype strains initiated a mean chemiluminescence of 9 SD $3 \%$ compared to the PMA control, and the rough morphotype strains $15 \mathrm{SD}$ $6 \%$ (Fig. 2). This difference was statistically significant $(p<0.005)$. However, no statistical difference between rough and smooth strains was found in the presence of serum (Fig. 3).

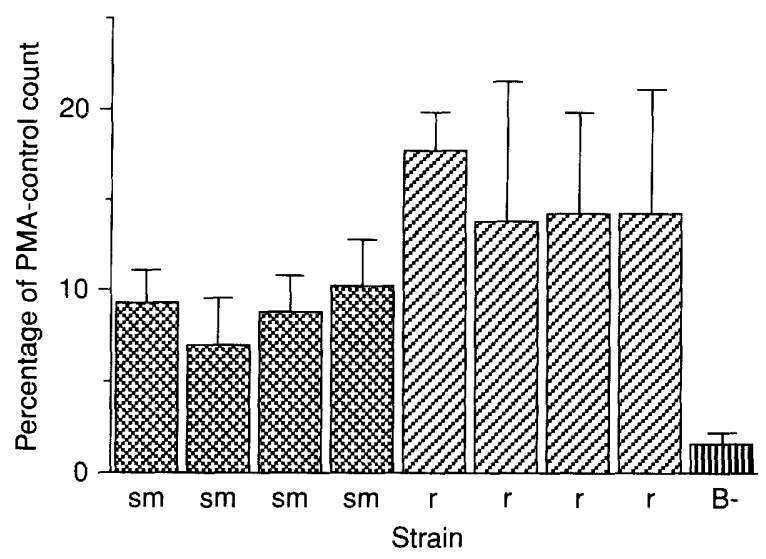

Fig. 2. Chemiluminescence of PMNLs induced by eight $P$. micros strains (mean and $\mathrm{SD} ; \mathrm{n}=4$ ) in the absence of serum. sm, smooth morphotype; r, rough morphotype; B - no bacteria added. Chemiluminescence counts were calculated as a percentage of the counts induced by a phorbol myristate acetate control.

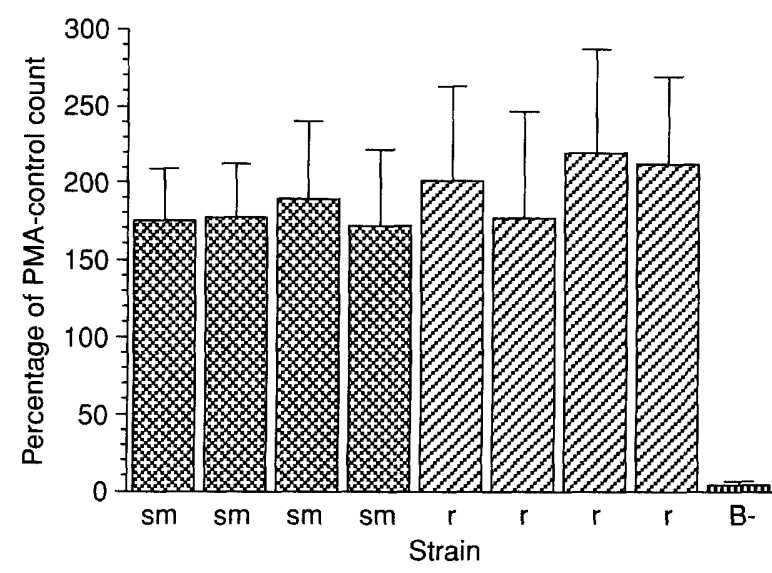

Fig. 3. Chemiluminescence of PMNLs induced by eight P. micros strains (mean and $\mathrm{SD} ; \mathrm{n}=7$ ) in the presence of pooled human serum. sm, smooth morphotype; r, rough morphotype; $\mathrm{B}-$, no bacteria added. Chemiluminesence counts were calculated as a percentage of the counts induced by a phorbol myristate acetate control.

\section{Discussion}

This paper reports on the pathogenicity of smooth and rough morphotype strains of $P$. micros. The most striking difference between the morphotypes of $P$. micros to date is the presence of fibrillar material on the surface of rough morphotype cells [3]. Further differences between types exist with respect to protein patterns of total cell lysates, hydrophobicity and haemolytic activity. The potential of $P$. micros to induce abscesses has been reported previously $[9,10,15,16]$, and the various experimental designs employed are varied with respect to the site and size of the inoculum and to the criteria for quantifying abscess formation. In all models, $P$. micros induced abscesses, usually containing fluid pus. Baumgartner et al. [15] reported an acute inflammatory reaction, characterised by mononuclear inflammatory cells and cellular debris in a welldefined abscess cavity. Abscesses induced by anaerobic gram-positive cocci usually drain after $10-15$ days [9, 10], leaving no visible sign of inflammation. In the current study the abscesses induced by the $P$. micros strains reached an optimum size 4 days after inoculation, with a small but significant difference in favour of the rough morphotypes. In general the diameter of the abscesses produced by $P$. micros morphotypes in this present model was smaller than those induced in the mouse groin by Brook et al. [10] which may be explained by the difference in the site of inoculum.

One representative strain of each morphotype was tested in further experiments to study their pathogenicity in combination with a strain of either $\mathrm{Pr}$. intermedia or Pr. nigrescens, as monocultures of these black-pigmented species generally do not induce transmissible infections [11]. In the combination experiments, the method of measuring the size of the abscesses was altered from one diagonal to two perpendicular diagonals and the contents were cultured. These changes made a more precise comparison between the strains possible. Low percentages of bacteria were recovered from the abscesses induced by all single-species inoculations of bacteria and when pus from these lesions was injected into other mice, lesions failed to develop. When both morphotypes of $P$. micros were combined, no increase of the abscess size was found and low numbers of both strains were recovered.

Pathogenic synergy between some of the species studied has been described before. Sundqvist et al. [9] showed that the presence of Pr. intermedia in mixed inocula was essential to cause transmissible abscesses in guinea-pigs. These mixed infections were transmissible, in spite of massive migration of PMNLs into the site of infection. In the current study pathogenic synergy between mixed cultures of either $P$. micros morphotypes and Pr. intermedia or $P r$. nigrescens was recorded. This synergy was characterised by transmissibility of infection, higher bacterial 
counts from pus and larger abscesses produced by mixed compared with single-species infections. The most effective combination was the rough morphotype of $P$. micros and $P r$. intermedia. In mixed infections involving peptostreptococci and gram-negative anaerobes, growth enhancement of the latter has been reported, whereas the growth rate of the peptostreptococci was not influenced by the gram-negative organisms [17]. This was also confirmed for the bacteria in the present experiments.

Possible important mechanisms in pathogenic synergy are nutritional inter-relationships and interactions with the host defence [11]. Ter Steeg et al. found that $P$. micros was the predominant micro-organism in batch-wise enrichment of subgingival plaque and $P r$. intermedia was one of the other species that survived in these experiments, irrespective of the origin of the samples [18]. These findings could be explained by a nutritional relationship between the species. Co-culture employed within the present study on various solid media (data not shown) and in broth revealed no evidence of nutritional interdependence between $P$. micros morphotypes or between $P$. micros strains and Prevotella strains. Although the nutritional situation in the gingival pocket is not comparable to these in-vitro assays, the data suggest that the pathogenic synergy described in the present study is not based on growth interactions. Possibly the explanation for this synergic activity can be related in part to the ability of the species in combination to evade the defence mechanisms of the host more effectively than the same species in monocultures. A possible mechanism is the mutual advantage conferred by immunoglobulin proteases produced by Prevotella spp. [19].

The interaction of $P$ micros with PMNLs was tested in the presence of human serum because this reflects the situation in vivo, but also in the absence of serum, because the opsonic capacity of the serum used may vary between morphotypes. In addition, in the absence of serum the induction of chemiluminescence may be more directly related to the surface structure of both $P$. micros morphotypes. The finding that the rough morphotype strains initiated higher counts than the smooth morphotype strains in the absence of serum might be related to the increased amount of fibrillar material on the surface of the rough morphotype cells [3]. It is possible that the fibrillar structures enable bacteria to adhere to PMNLs, which could have an effect also on their function. In the presence of serum the difference between the smooth and the rough morphotype strains may be masked by opsonisation of the cells.

In conclusion, the morphotypes of $P$. micros differ in virulence, with the rough morphotype being slightly more virulent in pure and in mixed infection with the two Prevotella species. However, further investigations are required to clarify the pathogenic mechanisms involved.

We thank W.F. Schouten (Department of Oral Microbiology, Vrije Universiteit, Amsterdam) for skilful assistance in the in-vivo experiments.

\section{References}

1. Murdoch DA, Mitchelmore IJ, Tabaqchali S. Peptostreptococcus micros from polymicrobial abscesses. Lancet 1988; 1: 594.

2. Rams TE, Feik D, Listgarten MA, Slots J. Peptostreptococcus micros in human periodontitis. Oral Microbiol Immunol 1992; 7: $1-6$.

3. van Dalen PJ, van Steenbergen TJM, Cowan MM, Busscher $\mathrm{HJ}$, de Graaff J. Description of two morphotypes of Peptostreptococcus micros. Int $J$ Syst Bacteriol 1993; 43: 787-793.

4. Shah HN, Gharbia SE. Biochemical and chemical studies on strains designated Prevotella intermedia and proposal of a new pigmented species, Prevotella nigrescens sp.nov. Int $J$ Syst Bacteriol 1992; 42: 542-546.

5. Johnson JL, Holdeman LV. Bacteroides intermedius comb. nov. and descriptions of Bacteroides corporis sp. nov. and Bacteroides levii sp. nov. Int J Syst Bacteriol 1983; 33: 15-25.

6. van Steenbergen TJM, Vlaanderen CA, de Graaff J. Deoxyribonucleic acid homologies among strains of Bacteroides melaninogenicus and related species. J Appl Bacteriol 1982; 53: $269-276$.

7. Dzink JL, Socransky SS, Haffajee AD. The predominant cultivable microbiota of active and inactive lesions of destructive periodontal diseases. $J$ Clin Periodontol 1988; 15: 316-323.

8. Jousimies-Somer H, Savolainen S, Mäkitie S, Ylikoski J. Bacteriologic findings in peritonsillar abscesses in young adults. Clin Infect Dis 1993; 16 Suppl 4: S292-S298.

9. Sundqvist GK, Eckerbom MI, Larsson ÅP, Sjögren UT. Capacity of anaerobic bacteria from necrotic dental pulps to induce purulent infections. Infect Immun 1979; 25: 685-693.

10. Brook I, Walker RI. Pathogenicity of anaerobic gram-positive cocci. Infect Immun 1984; 45: 320-324.

11. van Steenbergen TJM, van Winkenlhoff AJ, de Graaff J. Pathogenic synergy: mixed infections in the oral cavity. Antonie Van Leeuwenhoek 1984; 50: 789-798.

12. Brook I, Hunter V, Walker RI. Synergistic effect of Bacteroides, Clostridium, Fusobacterium, anaerobic cocci, and aerobic bacteria on mortality and induction of subcutaneous abscesses in mice. $J$ Infect Dis 1984; 148: 924-928.

13. Namavar F, Verweij AMJJ, Bal M, van Steenbergen TJM, de Graaff J, MacLaren DM. Effect of anaerobic bacteria on killing of Proteus mirabilis by human polymorphonuclear leukocytes. Infect Immun 1983; 40: 930-935.

14. van Steenbergen TJM, Namavar F, de Graaff J. Chemiluminescence of human leucocytes by black-pigmented Bacteroides strains from dental plaque and other sites. $J$ Periodont Res 1985; 20: $58-71$.

15. Baumgartner JC, Falkler WA, Beckerman T. Experimentally induced infection by oral anaerobic microorganisms in a mouse model. Oral Microbiol Immunol 1992; 7: 252-256.

16. Maiden MFJ, Lai CH, Tanner A. Characterization of oral gram-positive bacteria. In: Slots $\mathbf{J}$, Taubman MA (eds) Contemporary oral microbiology and immunology. St Louis, USA, Mosby Year Book. 1992: 342

17. Brook I. Enhancement of growth of aerobic, anaerobic, and facultative bacteria in mixed infections with anaerobic and facultative gram-positive cocci. J Surg Res 1988; 45: 222-227.

18. ter Steeg PF, Van der Hoeven J, de Jong MH, van Munster PJJ, Jansen MJH. Enrichment of subgingival microflora on human serum leading to accumulation of Bacteroides species, peptostreptococci and fusobacteria. Antonie Van Leeuwenhoek 1987; 53: 261-272.

19. Kilian M. Degradation of immunoglobulins A1, A2, and G by suspected principal periodontal pathogens. Infect Immun 1981; 34: $757-765$. 